\title{
Surgical techniques for early-stage thymoma: Video-assisted thoracoscopic thymectomy versus transsternal thymectomy
}

\author{
Bo Ye, MD, ${ }^{\mathrm{a}} \mathrm{Ji}-\mathrm{Cheng}$ Tantai, MD, ${ }^{\mathrm{a}}$ Xiao-Xiao Ge, MD, ${ }^{\mathrm{a}}$ Wang Li, MD,${ }^{\mathrm{b}}$ Jian Feng, MD, ${ }^{\mathrm{a}}$ \\ Ming Cheng, MD, ${ }^{\mathrm{a}}$ Jian-Xing Shi, $\mathrm{MD},{ }^{\mathrm{a}}$ and Heng Zhao, $\mathrm{MD}^{\mathrm{a}}$
}

Objective: The present study compared the outcomes between patients who had undergone video-assisted thoracoscopic surgery (VATS) thymectomy and transsternal (TS) thymectomy for Masaoka stage I and II thymoma.

\begin{abstract}
Methods: The outcomes of 262 patients without myasthenia gravis who had undergone surgery for Masaoka stage I and II thymoma from January 2008 to December 2012 at our center were retrospectively evaluated. The study included 125 patients who had undergone unilateral VATS thymectomy (VATS group) and 137 patients who had undergone TS thymectomy (TS group).
\end{abstract}

\begin{abstract}
Results: The VATS group had a shorter operative time than the TS group (170 vs 210 minutes, $P<.001)$. The VATS group also had a smaller intraoperative blood loss $(200 \mathrm{vs} 450 \mathrm{~mL}, P<.001)$, smaller pleural drainage volume in the first 24 hours postoperatively ( $300 \mathrm{vs} 500 \mathrm{~mL}, P<.0010$ ), shorter postoperative pleural drainage duration ( 3 vs 5 days, $P<.001$ ), and shorter postoperative hospital stay ( 8 vs 10 days, $P<.001$ ). Four patients in the VATS group underwent conversion to open surgery because of injury to the innominate vein. The postoperative complication rate was similar between the 2 groups. One patient in the VATS group developed pleural recurrence, and one in the TS group developed local recurrence.
\end{abstract}

Conclusions: Unilateral VATS thymectomy for Masaoka stage I and II thymoma is technically feasible and safe and is less invasive than TS thymectomy, with a shorter duration of surgery, less intraoperative blood loss, less postoperative pleural drainage, shorter postoperative pleural drainage duration, and shorter postoperative hospital stay. We have concluded that it is preferable to perform VATS thymectomy, although perhaps under certain circumstances sternotomy might be preferred. The oncologic outcomes were comparable between the 2 procedures. Additional follow-up is required to evaluate the long-term outcomes. (J Thorac Cardiovasc Surg 2014;147:1599-603)

Several studies have reported that video-assisted thoracoscopic surgery (VATS) thymectomy results in less operative trauma, a shorter hospital stay, better preservation of pulmonary function, and better cosmetic outcomes than transsternal (TS) thymectomy. ${ }^{1,2}$ However, it remains unclear whether VATS or TS thymectomy would be preferable in patients with noninvasive thymoma. ${ }^{3,4}$ A recent systematic review concluded that the current evidence regarding the long-term outcomes of minimally invasive surgery for thymoma is insufficient. ${ }^{5}$ The largest previously reported series comparing open and minimally invasive thymectomy included 77 patients who had undergone minimally invasive thymectomy, some of whom had undergone thymectomy for myasthenia gravis. ${ }^{3}$ No large series comparing the outcomes

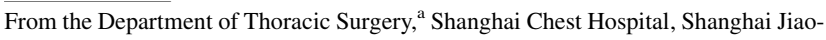
tong University, Shanghai, China; and Med-X Renji Clinical Stem Cell Research Center, ${ }^{\text {b }}$ Renji Hospital, Shanghai Jiao Tong University School of Medicine, Shanghai, China.

Disclosures: Authors have nothing to disclose with regard to commercial support.

Drs Ye and Tantai contributed equally to the present study.

Received for publication May 15, 2013; revisions received Sept 9, 2013; accepted for publication Oct 29, 2013; available ahead of print Dec 2, 2013.

Address for reprints: Heng Zhao, MD, Department of Thoracic Surgery, Shanghai Chest Hospital, Shanghai Jiaotong University, Huaihaixi Rd 241, Shanghai 200030, China (E-mail: 839974703@qq.com).

$0022-5223 / \$ 36.00$

Copyright (c) 2014 by The American Association for Thoracic Surgery

http://dx.doi.org/10.1016/j.jtcvs.2013.10.053
}

between VATS and TS thymectomy in patients without myasthenia gravis have been reported. The benefit of VATS thymectomy needs to be defined. The purpose of our study was to retrospectively compare the early outcomes of VATS and open TS thymectomy for stage I and II thymoma.

\section{METHODS \\ Patients}

The records of all 612 patients who had undergone resection of a primary thymic tumor with curative intent at Shanghai Chest Hospital from January 2008 to December 2012 were retrospectively reviewed. Of these, 304 patients with a pathologic diagnosis of Masaoka stage I thymoma (macroscopically and microscopically completely encapsulated) or stage II thymoma (microscopic transcapsular invasion, macroscopic invasion into the mediastinal fat, or grossly adherent to, but not through, the mediastinal pleura) ${ }^{6}$ had undergone unilateral VATS or TS thymectomy. A pathology specialist reviewed all the pathology data from the standard reports in the records. After exclusion of the 42 patients with myasthenia gravis, the remaining 262 patients (139 men and 123 women) were included in the present study. Of the 262 patients, 125 had undergone unilateral VATS thymectomy and 137 had undergone TS thymectomy. The characteristics of the patients in the VATS and TS groups are listed in Table 1. No significant differences were found between these 2 groups in age, gender, tumor stage, or the proportion of patients who underwent postoperative adjuvant radiotherapy. No patient received preoperative neoadjuvant therapy. No patient with Masaoka stage I thymoma received postoperative adjuvant therapy. All patients with stage II thymoma were offered postoperative adjuvant radiotherapy (total, $4000 \mathrm{cGy}$ ), but this 


\section{Abbreviations and Acronyms \\ TS $=$ transsternal \\ VATS $=$ video-assisted thoracoscopic surgery}

was refused by some patients. The study was conducted in accordance with the principles of the Declaration of Helsinki, and the ethics committee of Shanghai Chest Hospital, Shanghai Jiaotong University, approved the study protocol. All patients provided written informed consent for surgery. Because of the retrospective nature of the study, the requirement for patient consent for inclusion in the present study was waived.

\section{Operative Procedures}

Nine surgeons performed all the thymectomy procedures during the study period. These surgeons all performed both VATS and TS thymectomy. The choice of procedure was determined by the tumor characteristics and surgeon preference. TS thymectomy was performed in all patients with a tumor $>5 \mathrm{~cm}$ in diameter. Total thymectomy, with resection of all perithymic fat according to the Masaoka criteria, was performed in all patients. ${ }^{7}$

For unilateral VATS thymectomy, the patients were placed in the supine position under general anesthesia. The trachea was intubated with a doublelumen tube for split-lung ventilation. If the tumor was located centrally or on the right side, the right side of the chest was tilted upward by $30^{\circ}$. If the tumor was located on the left side, the left side of the chest was tilted upward by $30^{\circ}$. Surgery was performed through the side of the chest that was tilted upward, using a 3-port technique. A thoracoscope was introduced through a port in the fourth intercostal space at the posterior axillary line, at a $30^{\circ}$ oblique angle. Under direct vision, two 5-mm instrument ports were placed: one in the third intercostal space at the midaxillary line and the other in the sixth intercostal space at the anterior axillary line. In young female patients, the ports were sometimes placed in the submammary fold for cosmetic reasons. Dissection was performed as previously described. ${ }^{8}$ Starting on the side away from the tumor, care was taken to keep the plane of dissection beyond the capsule, to avoid direct traction to the capsule, and to limit capsular tears and local and pleural contamination by neoplastic cells. The dissection plane was extended cranially, taking care to avoid injury to the phrenic nerve. The left portion of the thymus was mobilized from the pericardium overlying the ascending aorta. The thymic veins were clipped and divided using ultrasonic or electrocautery dissection, and the right and left thymic horns were dissected. The specimen was removed in a plastic retrieval bag.

For TS thymectomy, the patients were placed in the supine position with the arms tucked in or slightly away from the body. The trachea was intubated with a single-lumen tube. Through complete or partial sternotomy, the thymus and tumor were resected en bloc. One chest tube was left in the patients of both groups, and the chest tube was removed when the chest tube drainage had been $<100 \mathrm{~mL}$ within 24 hours.

\section{Data Collection}

The surgery duration, intraoperative blood loss volume, pleural drainage volume within the first 24 hours after surgery, postoperative pleural drainage duration, postoperative hospital stay duration, and incidence of postoperative complications and recurrence were recorded. The tumor stage and tumor diameter were determined from the postoperative pathologic reports. Recurrence was determined from the follow-up chest contrast-enhanced computed tomography findings at 6 and 12 months after surgery and annually thereafter.

\section{Statistical Analysis}

The data are reported as the mean \pm standard deviation or median and range. Statistical analyses were performed using the Statistical Package for
Social Sciences, version 16.0, statistical software (SPSS, Inc, Chicago, Ill). The data were compared between the VATS and TS groups using the unpaired $t$ test, Fisher's exact test, or Mann-Whitney $U$ test, as appropriate. Propensity score matching using logistic regression analysis was performed to compare the groups after adjustment for gender, age, tumor stage, and adjuvant therapy.

\section{RESULTS}

The VATS group included 65 men and 60 women, with a mean age of 51.9 years and median follow-up period of 41 months (range, 1-60). The TS group included 74 men and 63 women, with a mean age of 50.0 years and median follow-up period of 42 months (range, 1-60). No patients were lost to follow-up during the study period.

In the VATS group, 90 patients underwent surgery through the right side and 35 through the left side. The perioperative outcomes of the patients in the VATS and TS groups are listed in Table 1 . The VATS group had a significantly shorter surgery duration, smaller intraoperative blood loss volume, smaller pleural drainage volume within 24 hours after surgery, shorter postoperative pleural drainage duration, and shorter postoperative hospital stay than the TS group $(P<.001$ for all $)$. Propensity score matching using logistic regression analysis showed that these variables all remained significantly different between the 2 groups after adjustment for gender, age, tumor stage, tumor size, and adjuvant therapy (Table 2).

Four patients in the VATS group underwent conversion to open surgery after injury to the left or right innominate vein, which caused massive bleeding in 3 cases. The characteristics of these 4 patients are listed in Table 3. Univariate logistic regression analyses identified only older age (odds ratio, $1.343 ; 95 \%$ confidence interval, $1.3053-1.712 ; P=.02$ ), larger tumor diameter (odds ratio, 1.277; 95\% confidence interval, 1.002-1.629; $P=.05$ ), and stage II disease (odds ratio, 6211; 95\% confidence interval, 5.714-6752000; $P=.01)$ as significant predictors of conversion to open surgery.

Postoperative complications included 3 cases of pneumonia, 1 case of pleural effusion, and 1 case of pulmonary atelectasis in the TS group and 1 case of pneumonia, 1 case of pleural effusion, 1 case of wound infection, and 3 case of pulmonary atelectasis in the VATS group. The difference in the complication rate between the 2 groups was not significant. In both groups, all surgical margins were free of tumor. No perioperative deaths occurred.

One patient in the VATS group developed pleural recurrence 42 months after surgery. This patient had had Masaoka stage II thymoma and had undergone postoperative adjuvant radiotherapy. One patient in the TS group developed local (pericardial) recurrence at 36 months after surgery. This patient had had Masaoka stage II thymoma and had initially refused postoperative adjuvant radiotherapy. These patients both underwent radiotherapy for 
TABLE 1. Characteristics and surgical outcomes

\begin{tabular}{|c|c|c|c|}
\hline Variable & VATS group & TS group & $P$ value \\
\hline Patients & 125 & 137 & \\
\hline Gender & & & $.805^{*}$ \\
\hline Male & 65 & 74 & \\
\hline Female & 60 & 63 & \\
\hline Age (y) & $51.9 \pm 13$ & $50 \pm 15.6$ & $.29 \dagger$ \\
\hline Masaoka stage & & & $.774 *$ \\
\hline I & 80 & 90 & \\
\hline II & 45 & 47 & \\
\hline \multicolumn{4}{|l|}{ WHO classification } \\
\hline A & 16 & 17 & \\
\hline $\mathrm{AB}$ & 85 & 92 & \\
\hline $\mathrm{B} 1$ & 17 & 23 & \\
\hline B2 & 4 & 3 & \\
\hline B3 & 3 & 1 & \\
\hline $\mathrm{C}$ & 0 & 1 & \\
\hline Tumor size $(\mathrm{mm})$ & $32.3 \pm 8.7$ & $33.8 \pm 6.5$ & $.063 \dagger$ \\
\hline Approach & & & - \\
\hline Right & 90 & - & \\
\hline Left & 35 & & \\
\hline Adjuvant RT (Masaoka II) & & & $.529^{*}$ \\
\hline Yes & $40 / 5$ & $41 / 6$ & \\
\hline No & & & \\
\hline Surgery duration (min) & & & $<.001 \ddagger$ \\
\hline Median & 170 & 210 & \\
\hline Range & $90-230$ & $130-350$ & \\
\hline Blood loss (mL) & & & $<.001 \ddagger$ \\
\hline Median & 200 & 450 & \\
\hline Range & $50-1500$ & $200-700$ & \\
\hline Postoperative pleural drainage (d) & & & $<.001 \ddagger$ \\
\hline Median & 3 & 5 & \\
\hline Range & $2-5$ & $3-7$ & \\
\hline Postoperative hospital stay (d) & & & $<.001 \ddagger$ \\
\hline Median & 8 & 10 & \\
\hline Range & $4-10$ & $5-32$ & \\
\hline Conversion to open surgery & 4 & - & \\
\hline Blood transfusion & 3 & 2 & $.672 *$ \\
\hline Postoperative complications & 6 & 5 & $.643^{*}$ \\
\hline 30-d Readmission & 9 & 10 & $.975^{*}$ \\
\hline Reintervention & 17 & 19 & $.950 *$ \\
\hline Follow-up (mo) & & & $.875 \ddagger$ \\
\hline Median & 41 & 42 & \\
\hline Range & $1-60$ & $1-60$ & \\
\hline Recurrence & 1 & 1 & 1 \\
\hline
\end{tabular}

their recurrence and were alive at the last follow-up examination but with additional disease progression.

\section{DISCUSSION}

Although TS thymectomy has been the traditional reference standard for the treatment of thymoma, ${ }^{9-13}$ VATS thymectomy has been increasing in popularity. At our institute, an increasing proportion of patients with

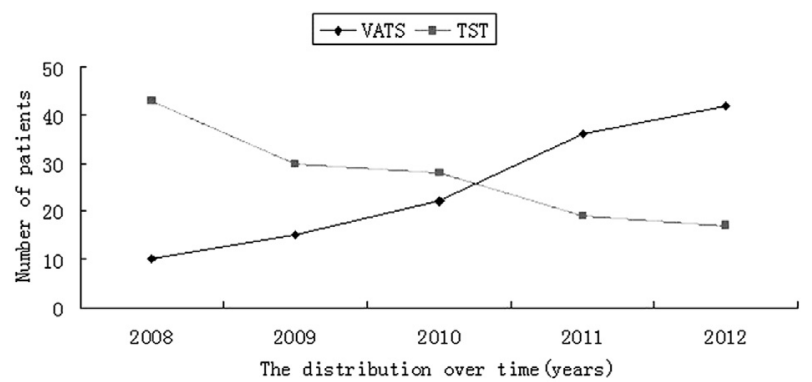

FIGURE 1. Patients who underwent video-assisted thoracoscopic thymectomy and transsternal thymectomy during each year of the study.

Masaoka stage I and II thymoma have been undergoing VATS thymectomy. This procedure has the advantage of being minimally invasive and provides an excellent view of the anterior mediastinum, allowing the surgeon to perform total thymectomy with resection of the perithymic fat. In the present series, the VATS group had a shorter surgery duration, smaller blood loss volume, smaller postoperative pleural drainage volume, shorter postoperative pleural drainage period, and shorter postoperative hospital stay than did the TS group. Furthermore, no serious postoperative complications occurred in the VATS group. These results indicate that unilateral VATS thymectomy is safe and feasible for the treatment of patients with stage I or II thymoma.

The optimal extent of surgical resection for the treatment of Masaoka stage I or II thymoma remains unclear. Some studies have concluded that complete resection of the tumor was desirable but did not distinguish between total thymectomy and partial thymectomy. ${ }^{14-16}$ Some studies have reported that complete resection of the tumor was sufficient for patients with Masaoka stage I and II thymoma who did not have myasthenia gravis. ${ }^{17,18}$ Wide resection of the thymus might increase the risk of surgical complications, and it is unclear whether wide resection is necessary in patients with small lesions. However, some reports have advocated total thymectomy because of the occasional development of recurrence. ${ }^{19,20}$ The incidence of multicentric tumor development has been estimated to be about $2 \% .{ }^{21}$ It will usually be easy to diagnose the recurrence of thymoma using computed tomography, and patients with recurrence in the residual thymus should undergo completion thymectomy. ${ }^{22}$ Completion thymectomy can be more safely performed after thoracoscopic surgery than after sternotomy. ${ }^{23}$

Only 4 patients in the VATS group underwent conversion to open surgery. These 4 patients had all undergone surgery during 2008 or 2009 and required conversion to open surgery after injury to the left or right innominate vein caused by electrocautery dissection. The drainage veins from the thymus to the innominate vein are thin vessels, and we now carefully isolate and clip each vessel and use ultrasonic 


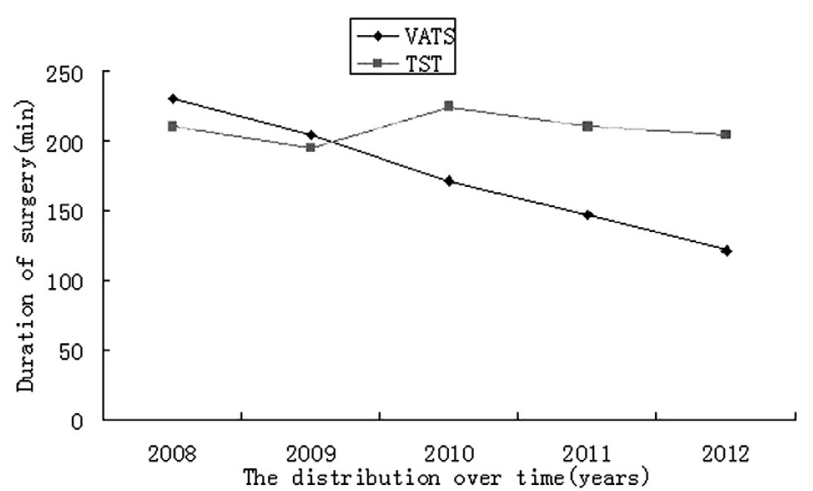

FIGURE 2. Median duration of surgery in patients who underwent videoassisted thoracoscopic thymectomy and transsternal thymectomy during each year of the study.

coagulation to minimize bleeding. Despite the massive bleeding in 3 of these patients, the intraoperative blood loss was significantly less in the VATS group than in the TS group.

In our experience, VATS thymectomy allowed safe resection without damage to the thymoma capsule. However, the size of the lesion will probably affect the success of the minimally invasive approach. In the present series, the mean diameter of the thymoma was $32.3 \pm 8.7 \mathrm{~mm}$ in the VATS group and $33.8 \pm 6.5 \mathrm{~mm}$ in the TS group, similar to the size reported in other series comparing these procedures. $^{8,24}$

VATS thymectomy has limitations but relatively few contraindications. In addition to the general contraindications such as severe coagulopathy, the specific contraindications include pleural adhesions and patients with severe underlying lung disease or poor lung function who are unable to tolerate selective single-lung ventilation. ${ }^{1}$ VATS might not be an ideal approach for very young children because of the difficulty of performing single-lung ventilation in this age group. ${ }^{1}$

The long-term oncologic outcomes after VATS thymectomy for thymoma remain unclear. Some studies have reported tumor spread to the chest cavity after surgery. ${ }^{21,25}$ A comparative study of 40 patients by Pennathur and

TABLE 2. Adjusted surgical outcomes for potentially confounding factors

\begin{tabular}{llll}
\hline \multicolumn{1}{c}{ Variable } & VATS group & \multicolumn{1}{c}{ TS group } & $\boldsymbol{P}$ value \\
\hline Blood loss (mL) & $183.1 \pm 98.2$ & $462.4 \pm 95.7$ & $<.001$ \\
Surgery duration (min) & $171.0 \pm 31.1$ & $216.0 \pm 41.2$ & $<.001$ \\
$\begin{array}{l}\text { Postoperative pleural } \\
\quad \text { drainage within 24 h (mL) }\end{array}$ & $311.8 \pm 122.0$ & $496.6 \pm 112.7$ & $<.001$ \\
$\begin{array}{l}\text { Postoperative pleural } \\
\quad \text { drainage duration (d) }\end{array}$ & $3.2 \pm 0.8$ & $4.9 \pm 0.9$ & $<.001$ \\
Postoperative hospital stay (d) & $7.3 \pm 1.5$ & $12.36 \pm 7.6$ & $<.001$ \\
\hline
\end{tabular}

$P$ value by covariance analysis. VATS, Video-assisted thoracoscopic (thymectomy); $T S$, transsternal (thymectomy).
TABLE 3. Characteristics of 4 patients who required conversion from VATS to open surgery

\begin{tabular}{lc}
\hline \multicolumn{1}{c}{ Variable } & Value \\
\hline Age (y) & $52.8 \pm 7.9$ \\
Gender & \\
Male & 2 \\
Female & 2 \\
Approach & \\
Right & 1 \\
Left & 3 \\
Tumor size (mm) & $40 \pm 8.2$ \\
Masaoka stage & \\
I & 1 \\
II & 3 \\
Surgery duration (min) & $205 \pm 20.8$ \\
Blood loss (mL) & $825 \pm 478.7$ \\
Blood transfusion (mL) & \\
Patient 1 & 800 \\
Patient 2 & 400 \\
Patient 3 & 400 \\
Patient 4 & 0 \\
Postoperative pleural & \\
$\quad$ drainage within 24 h (mL) & \\
Postoperative pleural & $450 \pm 129.1$ \\
$\quad$ drainage (d) & \\
Postoperative hospital stay (d) & $4.5 \pm 0.6$ \\
VATS, Video-assisted thoracoscopic surgery. & $6.8 \pm 2.2$ \\
\hline
\end{tabular}

VATS, Video-assisted thoracoscopic surgery.

colleagues $^{8}$ reported no significant differences in disease recurrence or overall survival between the VATS and TS groups after a mean follow-up period of 36 months. Cheng and colleagues ${ }^{26}$ observed no local or pleural recurrence after resection of stage II thymoma in either the VATS or TS group. A recent systematic review of surgical resection for thymoma reported a 5 -year survival rate of $89 \%$ to $100 \%$ in patients with stage I thymoma and $71 \%$ to $95 \%$ in patients with stage II thymoma. In the present series, 1 patient developed pericardial recurrence and 1 developed pleural recurrence; both patients were still alive at the latest follow-up visit. The oncologic outcomes were, therefore, comparable between the VATS and TS groups in the intermediate term, consistent with previously reported results. However, thymoma requires long-term follow-up because of the slowgrowing nature of the disease. ${ }^{19}$ Additional studies with larger numbers of patients and $\geq 10$ years of follow-up are required to evaluate the long-term survival and diseasefree rates.

\section{Study Limitations}

The present study was limited by its retrospective singlecenter design and relatively short follow-up period. The surgical procedure performed and the decision to convert to open surgery were at the discretion of the surgeon, which might have resulted in selection bias. The team of surgeons who performed the operations was quite large and was a 
bias of the present study. Our series had a limited number of cases, and the follow-up duration was not long enough. These preliminary results of our series suggest that VATS for Masaoka stage I and II thymoma is technically feasible and safe and is less invasive for the patient. Nevertheless, this procedure requires additional investigation in a large series with longer follow-up. More cases series should be investigated to establish the validity of our procedure.

However, our findings are important, because this is the largest reported series comparing unilateral VATS thymectomy with TS thymectomy for the treatment of Masaoka stage I or II thymoma in patients without myasthenia gravis.

\section{CONCLUSIONS}

The results of the present study have shown that unilateral VATS thymectomy is safe and feasible for the treatment of Masaoka stage I or II thymoma. VATS thymectomy was associated with a shorter postoperative hospital stay, less intraoperative blood loss, less postoperative pleural drainage, and shorter postoperative pleural drainage duration than TS thymectomy. We have concluded that it is preferable to perform VATS thymectomy, although perhaps under certain circumstances sternotomy might be preferred. The intermediate-term oncologic outcomes were similar between the VATS and TS thymectomy groups. Additional follow-up is required to evaluate the long-term outcomes of VATS thymectomy for early-stage thymoma.

\section{References}

1. Ng CS, Wan IY, Yim AP. Video-assisted thoracic surgery thymectomy: the better approach. Ann Thorac Surg. 2010;89:\$2135-41.

2. Odaka M, Akiba T, Yabe M, Hiramatsu M, Matsudaira H, Hirano J, et al. Unilateral thoracoscopic subtotal thymectomy for the treatment of stage I and II thymoma. Eur J Cardiothorac Surg. 2010;37:824-6.

3. Jurado J, Javidfar J, Newmark A, Lavelle M, Bacchetta M, Gorenstein L, et al. Minimally invasive thymectomy and open thymectomy: outcome analysis of 263 patients. Ann Thorac Surg. 2012;94:974-82.

4. Yoshida S, Yoshino I, Moriya Y, Hoshino H, Okamoto T, Suzuki M, et al. Videoassisted thoracoscopic surgery extended thymectomy for myasthenia gravis using manual manipulators: the radius surgical system. Ann Thorac Surg. 2011; 92:2246-8.

5. Davenport E, Malthaner RA. The role of surgery in the management of thymoma: a systematic review. Ann Thorac Surg. 2008;86:673-84.

6. Masaoka A. Staging system of thymoma. J Thorac Oncol. 2010;5:S304-12.
7. Masaoka A, Yamakawa Y, Niwa H, Fukai I, Kondo S, Kobayashi M, et al. Extended thymectomy for myasthenia gravis patients: a 20-year review. Ann Thorac Surg. 1996;62:853-9.

8. Pennathur A, Qureshi I, Schuchert MJ, Dhupar R, Ferson PF, Gooding WE, et al. Comparison of surgical techniques for early-stage thymoma: feasibility of minimally invasive thymectomy and comparison with open resection. J Thorac Cardiovasc Surg. 2011;141:694-701.

9. Roviaro G, Varoli F, Nucca O, Vergani C, Maciocco M. Videothoracoscopic approach to primary mediastinal pathology. Chest. 2000;117:1179-83.

10. Yim AP. Video-assisted thoracoscopic resection of anterior mediastinal masses. Int Surg. 1996;81:350-3.

11. Takeo S, Fukuyama S. Video-assisted thoracoscopic resection of a giant anterior mediastinal tumor (lipoma) using an original sternum-lifting technique. Jpn J Thorac Cardiovasc Surg. 2005;53:565-8.

12. Sugarbaker DJ. Thoracoscopy in the management of anterior mediastinal masses Ann Thorac Surg. 1993;56:653-6.

13. Roviaro G, Rebuffat C, Varoli F, Vergani C, Maciocco M, Scalambra SM. Videothoracoscopic excision of mediastinal masses: indications and technique. Ann Thorac Surg. 1994;58:1679-84.

14. Kohman LJ. Controversies in the management of malignant thymoma. Chest 1997;112:296S-300S.

15. Okumura M, Ohta M, Tateyama H, Nakagawa K, Matsumura A, Maeda H, et al The World Health Organization histologic classification system reflects the oncologic behavior of thymoma: a clinical study of 273 patients. Cancer. 2002;94: 624-32.

16. Moore KH, McKenzie PR, Kennedy CW, McCaughan BC. Thymoma: trends over time. Ann Thorac Surg. 2001;72:203-7.

17. Sakamaki Y, Kido T, Yasukawa M. Alternative choices of total and partial thymectomy in video-assisted resection of noninvasive thymomas. Surg Endosc. 2008;22:1272-7.

18. Singhal S, Shrager JB, Rosenthal DI, LiVolsi VA, Kaiser LR. Comparison of stages I-II thymoma treated by complete resection with or without adjuvant radiation. Ann Thorac Surg. 2003;76:1635-42.

19. Awad WI, Symmans PJ, Dussek JE. Recurrence of stage I thymoma 32 years after total excision. Ann Thorac Surg. 1998;66:2106-8.

20. Murakawa T, Nakajima J, Kohno T, Tanaka M, Matsumoto J, Takeuchi E, et al. Results from surgical treatment for thymoma: 43 years of experience. Jpn J Thorac Cardiovasc Surg. 2000;48:89-95.

21. Bernatz PE, Harrison EG, Clagett OT. Thymoma: a clinicopathologic study J Thorac Cardiovasc Surg. 1961;42:424-44.

22. Kirschner PA. Reoperation on the thymus: a critique. Chest Surg Clin North Am 2001;11:439-45.

23. Pompeo E, Nofroni I, Iavicoli N, Mineo TC. Thoracoscopic completion thymectomy in refractory nonthymomatous myasthenia. Ann Thorac Surg. 2000;70: 918-23.

24. Cheng YJ, Wu HH, Chou SH, Kao EL. Video-assisted thoracoscopic management of mediastinal tumors. JSLS. 2001;5:241-4.

25. Chen G, Marx A, Chen WH, Yong J, Puppe B, Stroebel P, et al. New WHO histologic classification predicts prognosis of thymic epithelial tumors: a clinicopathologic study of 200 thymoma cases from China. Cancer. 2002;95: 420-9.

26. Cheng YJ, Kao EL, Chou SH. Videothoracoscopic resection of stage II thymoma: prospective comparison of the results between thoracoscopy and open methods. Chest. 2005;128:3010-2. 